\title{
STRUCTURE CONSTRAINTS FROM LARGE ANGLE CMB ANISOTROPIES
}

\author{
J. Richard Bond \\ CIAR Cosmology Program, Canadian Institute for Theoretical Astrophysics \\ Univesity of Toronto, ON MSS 1A1, Canada
}

Constraints on models of cosmic structure formation that can be drawn from current limits on large angle microwave background anisotropies are now competitive with those from recent small and intermediate angle experiments and are relatively insensitive to the reheating history of the Universe. Here I give limits on Gaussian scale invariant adiabatic fluctuations and describe the role that the large angle results play in constraining models with enhanced large distance galaxy clustering power inferred from correlation function measurements is described.

The main paradigm for galaxy formation throughout the 1980's was the inflationinspired theory in which, within the patch of the Universe accessible to our observations (approximately the Hubble volume $\left[\mathrm{cH}_{0}^{-1}\right]^{3}$ ), the mean curvature $\left\langle{ }^{(3)} R\right\rangle$ is tiny, $\left(^{(3)} R\right\rangle \lesssim 10^{-4}\left[\mathrm{cH}_{0}^{-1}\right]^{-2}$. To have larger mean curvature is to invite strong curvature fluctuations which lead to unacceptable large angle anisotropies or to demand a huge rise in the curvature fluctuations on scales just larger than our horizon. Thus $\Omega \approx 1$. Together with the primordial nucleosynthesis constraint on the baryon density parameter $\Omega_{B}, \lesssim 0.064\left(50 / H_{0}\right)^{2}$, a great deal of dark matter that was not baryonic during the first 3 minutes is necessary, whether it be cold, warm or hot dark matter relics, nonzero vacuum energy (i.e., nonzero cosmological constant), slowly decreasing scalar field energy, relativistic decay products, or some combination of the above. There is evidence from the IRAS survey that the $\Omega$ in matter which clusters is not too far off unity. Since vacuum (or $\Lambda$ ) energy does not cluster, non-zero $\Lambda$ becomes at least as unpalatable as any of the dark matter hybrid models, such as hot/cold, with the added conundrum that the length $\Lambda^{-1 / 2}$ exceeds $10^{60}$ Planck lengths, while the Planck length is its apparent natural unit.

Although minimalism may not be an operating principle in our patch of the Universe, it gives a good base from which to add on extra ingredients. With the $\Omega_{B}$ constraint and the IRAS estimate and the need for dark matter clustering in dwarf galaxies, the minimal assumption for dark matter is that it is cold (CDM). The most conservative assumption for the fluctuations that arise in the early Universe is that they have no built-in scale (are scaleinvariant) and that the power they have is shared as democratically as possible - that is, in a maximally random fashion (are Gaussian-distributed) and that they are perturbations in the curvature (are adiabatic). If isocurvature perturbations dominate, there must also be a curvature-perturbation suppression-mechanism, hence non-minimality.

Inflation models with one dynamically important scalar field invariably give fluctuations which are Gaussian-distributed within Hubble volume scales (although are wildly non-Gaussian on much much larger scales) and are nearly scale invariant and are adiabatic. With cold dark matter, this defines the 'standard' CDM model, which is, in this sense, minimal. Globular cluster age constraints force us to take $H_{0} \lesssim 50$, and the persistent reports of higher values is the Achilles heel of minimal-CDM-ism, forcing upon us a non-zero $\Lambda$ or a slowly decaying scalar field to get the time-span up.

With scale invariance, the $r m s$ gravitational potential fluctuations $\sigma_{\Phi}$ initially have equal contributions per decade of wavenumber: i.e., $d \sigma_{\Phi}^{2} / d \ln k \approx\left(2 \times 10^{-5} \sigma_{8}\right)^{2}$ is inde- 
pendent of wavenumber, depending upon a constant normalization parameter $\sigma_{8}$ in the manner indicated. (In a chaotic inflation model with a potential $V(\phi)=\lambda \phi^{4} / 4$ we have $\lambda \approx 6 \times 10^{-14} \sigma_{8}^{2}$. How to have such a weak coupling is the fine tuning problem of inflation.)

More generally, for any spectrum we define $\sigma_{8}$ to be the $r m s$ amplitude of mass density fluctuations on the scale $8 \mathrm{~h}^{-1} \mathrm{Mpc}$ at the present time (using linear perturbation theory - a convention). [ $\left.\mathrm{h}=H_{0} / 100\right]$. In particular, I shall discuss power law inflation models, for which $d \sigma_{\Phi}^{2} / d \ln k \approx\left(2 \times 10^{-5} \sigma_{8}\right)^{2}\left(k^{-1} / 5 \mathrm{~h}^{-1} \mathrm{Mpc}\right)^{\left(1-n_{s}\right)}$, where $n_{s}$ gives the initial slope of the spectrum. Thus $n_{s}=1$ gives scale-invariance (Harrison-Zeldovich). If the inflaton potential $V(\phi)$ looks like an exponential over some stretch of $\phi$, then $n_{s}$ will be less than one. In natural inflation, which explains the tiny $\lambda$ dilemma in terms of a ratio $\left(M_{G U T} / m_{P l a n c k}\right)^{4}$, an approximate power law is also expected (Freese et al. 1991).

Anisotropies on scales above a few degrees can provide a direct window on the primordial fluctuations in the geometry of spacetime. Anisotropies on smaller angular scales probe as well gas dynamics at the time of photon decoupling, and their predicted values are sensitive to assumptions about the reionization history of the Universe. Thus although the Owens Valley experiment (Readhead et al. 1989), with a $1.8^{\prime}$ beam, and the South Pole experiment of Meinhold and Lubin, with its $30^{\prime}$ beam, provide powerful constraints (see Bond et al. 1991, BELM) if the Universe underwent normal recombination and did not reionize until well below redshift 100 , experiments with beams of order a few degrees are better for probing Universes with reionization above a few hundred and experiments with beams above about $7^{\circ}$ probe curvature fluctuations only.

For initial power law models with spectral index $n_{s}$ which are dominated by CDM (or HDM), curvature fluctuations give a Sachs-Wolfe contribution to the rms anisotropies in the $2^{L}$ - multipole ) of the $\Delta T / T$ pattern which, for $L \lesssim 20$ or so, is simply expressible in an analytic form:

$$
\left\langle[\Delta T / T]_{L}^{2}\right\rangle=\frac{(2 L+1)}{5} \frac{\left[L-\frac{\left(3-n_{s}\right)}{2}\right] !}{\left[L+\frac{\left(3-n_{s}\right)}{2}\right] !} \frac{\left[\frac{\left(7-n_{s}\right)}{2}\right] !}{\left[\frac{\left(1+n_{s}\right)}{2}\right] !}\left\langle[\Delta T / T]_{\ell=2}^{2}\right\rangle .
$$

(Thus for scale invariance $\left(n_{s}=1\right.$ ), the angular power spectrum, $\left\langle[\Delta T / T]_{L}^{2}\right\rangle d L$ has equal power per decade of $L, \sim d L / L$, just as the 3D spectrum does per decade of $k$.)

For an experiment like COBE's DMR, this should be multiplied by its approximately Gaussian beam filter, $\exp \left[-(L+0.5)^{2} /\left(L_{s}+0.5\right)^{2}\right]$, where $L_{s} \approx 20$ corresponds to their fwhm beam size of $7^{\circ}$. For $n_{s}=1$ and $\Omega_{B}=0$, we have a quadrupole amplitude $\left\langle[\Delta T / T]_{\ell=2}^{2}\right\rangle^{1 / 2} \approx 0.4 \times 10^{-5} \sigma_{8}$ and $r m s$ anisotropies on the scale of the COBE beam of $\left\langle[\Delta T / T]^{2}\left(7^{o}\right)\right\rangle^{1 / 2} \approx 10^{-5} \sigma_{8}$. (This rises by about $20 \%$ as $\Omega_{B}$ increases to 0.1 .)

$\begin{array}{llccccc} & \Omega_{B} & \text { DMR }\left(7^{\circ}\right) & \text { RELICT } & \text { SPole+OVRO } & \text { SPole } & \text { OVRO } \\ \text { SR } & 0.01 & 3.7 & 3.6 & 1.8 & 2.3 & 3.3 \\ \text { SR } & 0.03 & 3.6 & 3.5 & 1.4 & 2.1 & 2.2 \\ \text { SR } & 0.1 & 3.1 & 3.0 & 1.0 & 1.5 & 1.5 \\ \text { NR } & 0.1 & 2.6 & 3.0 & 3.4 & 3.4 & 17\end{array}$

In this table, we list the $95 \%$ credible limits derived by BELM on $\sigma_{8}$ for standard CDM models as a function of $\Omega_{B}$, for OVRO data alone, the Meinhold and Lubin South Pole data alone, and for both combined. Here, SR denotes standard recombination and NR denotes no recombination, which is a limiting case of early reionization. We compare it with the constraints for RELICT 1 (Klypin et al. 1987) and DMR (Smoot et al. 1991). The RELICT limit was obtained assuming scale invariance, and the differences reflect only the $\Omega_{B}$ dependence of the spectrum normalization. The Boughn et al. (1991) limits give 
values similar to those of DMR. Preliminary results for the MIT balloon experiment of Meyer et al. (1991) (beam of $3.8^{\circ}, L_{s} \sim 30$ ) indicate a factor of about two improvement over the RELICT limits is possible.

The strongest evidence that the CDM model has too little power at large scales comes from the angular correlation function $w_{g g}(\theta)$ of galaxies. Proposed solutions and the influence of the CMB limits on these include:

(1) CDM plus non-local biasing plus nonlinear dynamics. Carlberg and Couchman (1991) showed that a $\sigma_{8}=1.25$ model could explain the data, but this is already under pressure from $\Delta T / T$ constraints. However, modifications in the non-local biasing scheme could explain the extra power, without requiring that $\sigma_{8}$ be so high.

(2) CDM plus non-Gaussian statistics. This can arise naturally in models with topological defects such as strings and global monopoles and textures. The fluctuations are isocurvature, the spectrum is (likely to be) scale invariant, but $\Delta T / T$ calculations which confront the data are difficult because the initial conditions must be simulated on a lattice, with the size restrictions from computer memory limitations that entails.

(3) CDM plus broken scale invariance of the power law form. The angular correlation data suggests $0 \lesssim n_{s} \lesssim 0.4$, but the RELIC $T$ experiment suggests that $n_{s} \gtrsim 0.6$ for $\sigma_{8}=1$ and $n_{s} \gtrsim 0.3$ for $\sigma_{8}=0.5$. Other spectral forms which can arise are two flat spectra joined by a ramp crafted to fit $w_{g g}$ (double inflation can give this but it runs afoul of the large angle limits) or mountain and valley spectra, which can be crafted to evade the large angle constraints but cannot evade the small angle constraints unless there is early reionization. To get the sharp features needed for these mountain and valley spectra requires sudden changes on the potential surface constructed to appear just at the length scales our large scale structure observations are probing, $k^{-1} \sim(5-50) \mathrm{h}^{-1} \mathrm{Mpc}$.

(4) Assume CDM and add more constituents to the Universe. Two possibilities along these lines are: The introduction of nonzero $\Lambda$ so that $\left(\Omega_{c d m}+\Omega_{B}\right) \approx 0.4\left(50 / H_{0}\right)$. With $H_{0}=50$ these models are ruled out (BELM), but for $H_{0}=100$ they are still viable. The addition of a $17 \mathrm{kev}$ massive neutrino (reported in a variety of beta decay experiments with solid state detectors). It must decay to avoid cosmological catastrophes. If it decays with an appreciable branching ratio to radiative decay, it must do so with a lifetime less than a month or it over-distorts the CMB. If it primarily decays into non-radiative channels then if it has a lifetime in the 1-10 year range it can explain $w_{g g}$. However, for $\sigma_{8}=1$, we must have a lifetime less than a year and for $\sigma_{8}=0.5$ less than 5 years (Bond and Efstathiou 1991). Other strategies for gaining extra power are discussed in BBE.

Modest improvements in the large angle limits such as those inferred for the MIT balloon experiment and those expected from DMR may rule out many of these extra power models and could put the minimal model under severe stress even with early reionization.

Bardeen, J.M., Bond, J.R. and Efstathiou, G. 1987, Ap. J., 321, 28 [BBE].

Bond, J.R., Efstathiou, G., Lubin, P.M. and Meinhold, P.R. 1991, Phys. Rev. Lett., 66,

2179 [BELM]; see also Vittorio, N. et al. 1991, Ap. J. Lett., 372, L1.

Bond, J.R. and Efstathiou, G. 1991, Phys. Lett. B 265, 245.

Boughn, S., Cheng, E., Cottingham, D. and Fixsen, D. 1991, Ap. J. Lett., preprint. Carlberg, R.G. and Couchman, H.M.P. 1991, preprint.

Freese, K., Frieman, J. and Olinto, A. 1991, preprint.

Klypin, A., Sazhin, M., Strukov, I. and Skulachev, D. 1987, Sov. Astr. Lett., 13, 104.

Meyer, S.S., Cheng, E.S. and Page, L.A. 1991, Ap. J. Lett., 371, L7.

Readhead, A.C.S. et al. 1989, Ap. J., 346, 566.

Smoot, G. et al. 1991, Ap. J. Lett., 371, L1. 